\title{
La comunicación digital de la empresa de franquicia: uso y presencia en los medios sociales
}

\author{
Araceli Castelló Martínez \\ Departamento de Comunicación y Psicología Social. \\ Facultad de Ciencias Económicas y Empresariales. Universidad de Alicante \\ Juan Monserrat Gauchi \\ Departamento de Comunicación y Psicología Social. \\ Facultad de Ciencias Económicas y Empresariales. Universidad de Alicante
}

\section{Palabras clave}

Marketing, comunicación, publicidad, Internet, medios sociales, franquicia

\section{Resumen}

El artículo se centra en el estudio de la presencia de la empresa de franquicia en los medios sociales, analizando las estrategias de comunicación empresarial y publicitaria que llevan a cabo en plataformas como Facebook o Twitter. Se parte de la hipótesis de que la empresa franquicia todavía tiene escasa presencia en los espacios 2.0 y que, en general, se limita a volcar información de otros canales, generando poca conversación con sus seguidores. La metodología se basa, por una parte, en la investigación documental de publicaciones de los ámbitos profesional y académico, repasando la situación actual de los medios sociales, y en un estudio exploratorio a partir del análisis de case studies de las principales empresas de franquicia, por otra. Los resultados del estudio nos obligan a hablar del escaso aprovechamiento que la empresa franquicia está haciendo de las bondades de las plataformas 2.0, por lo que se cumple la hipótesis de partida. El crecimiento experimentado por estos espacios en los últimos años y las ventajas que, por las peculiaridades de la empresa franquicia, el Social Media Marketing puede aportar a su estrategia de comunicación y publicidad, obligan al desarrollo de una estrategia clara y definida desde la central de franquicia que determine la presencia de cada uno de los franquiciados en las redes sociales. 


\title{
Franchise company digital communication: use and presence on social media
}

\section{Keywords}

Marketing, communication, advertising, Internet, social media, franchise

\begin{abstract}
The article focuses on the study of franchise company presence on social media, analyzing business and advertising communication strategies that it carries out in platforms such as Facebook or Twitter. It starts from the hypothesis that franchise company still has limited presence on 2.0 spaces and that, in general, it is just confined to dump information from other channels, barely generating conversation with its followers. The method is based on documentary research of both, academic and professional publications, reviewing current situation of social media, on the one hand, and the analysis of case studies of major franchise companies, on the other. The results of the study compel us to talk about the limited use that franchise company is making of the benefits of 2.0 platforms; therefore, the initial hypothesis is demonstrated. The growth experienced by these channels in recent years and the advantages that, due to the franchise company peculiarities, Social Media Marketing can bring to its communication and advertising strategy, require the development of a clear and defined strategy from the franchise central that determines each of the franchisees presence on social media.
\end{abstract}

\section{Autores}

Araceli Castelló Martínez [araceli.castello@ua.es] es profesora en la Universidad de Alicante de la asignatura Introducción a la investigación de medios y colaboradora en diversos postgrados. Es doctora en Comunicación, magíster en Comunicación Integral y licenciada en Publicidad y RR.PP. Ha publicado el libro Estrategias empresariales en la Web 2.0, así como informes, artículos y capítulos sobre los medios sociales y la comunicación corporativa y publicitaria online.

Juan Monserrat Gauchi [Juan.Monserrat@ua.es] es profesor colaborador doctor en la Universidad de Alicante. Responsable de la asignatura Sistemas y procesos en Publicidad y Relaciones Públicas. Realizó su tesis doctoral sobre los modelos comunicativos en las empresas de franquicia y, ésta es un de sus principales líneas de investigación. Ha sido profesor invitado por la Universidad Nacional Autónoma de México y por The California State University en 2011. 


\section{Introducción}

En 2007 la publicación Advertising Age vaticinaba que «las fronteras del marketing entre los formatos digitales y los tradicionales se difuminarán definitivamente a lo largo de los próximos cinco años». Es lo que autores como Alonso Coto (2008: 5) llaman blended marketing, al defender que el plan de marketing actual es digital, al integrar las nuevas posibilidades de las herramientas online con formatos y medios tradicionales, combinación que refuerza los valores de marca:

«Es clave integrar estas nuevas posibilidades de marketing digital con los formatos y medios que llevan años demostrando su eficacia. De hecho, el uso combinado de herramientas online y offline en una misma campaña de marketing, potencia el recuerdo de los valores de marca en hasta un $18 \%$, lo que refrenda a Internet como un medio muy rentable e inexcusable pero en ningún caso exclusivo para toda campaña de marketing, comunicación y publicidad».

La saturación publicitaria y la profesionalización de los hábitos de navegación del usuario, entre otros, han motivado que cada vez más los esfuerzos de los anunciantes se centren en espacios en los que la marca y el producto se integran con el contenido y, más allá, la marca crea su propio contenido para compartirlo con los usuarios (brand content), como sucede en los medios sociales.

Plataformas 2.0 como Facebook o Twitter han ampliado el rango de posibilidades que ofrece la red como soporte para la comunicación corporativa y publicitaria, sobre todo gracias a espacios en los que el cliente forma parte de la comunidad de la marca y conversa con la empresa anunciante, en contraposición con la unidireccionalidad que tradicionalmente ha caracterizado a los medios convencionales como canales publicitarios:

\section{Cuadro 1. Social Engagement Spectrum ${ }^{1}$}

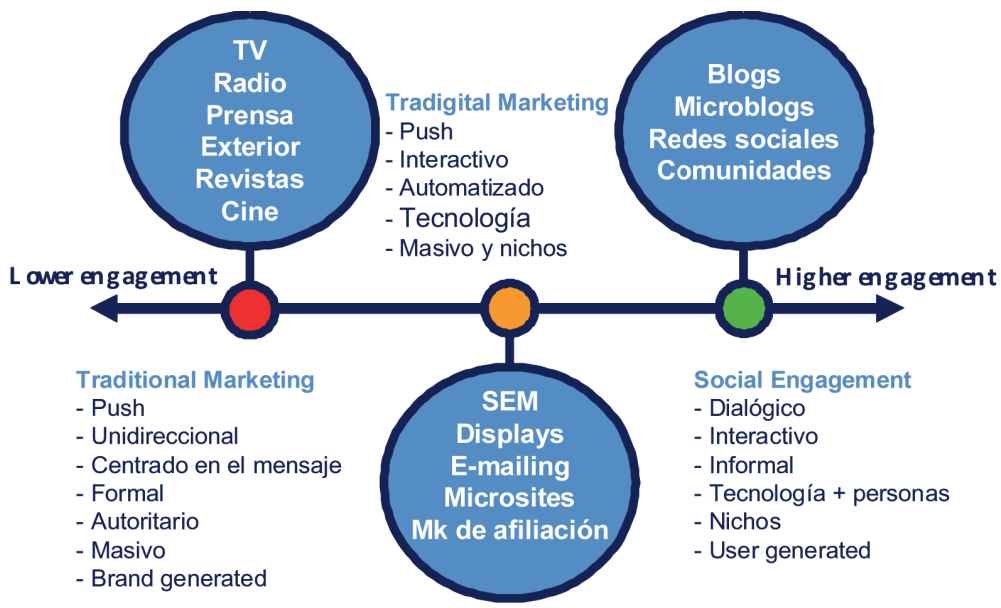

1 Adaptado de David Armano: http://darmano.typepad.com/logic_emotion/2009/05/social-engagement-spectrum.html 
El informe Marketing en medios sociales publicado por Territorio Creativo en abril de 2010 destaca que ocho de cada diez empresas que llevan a cabo acciones de Social Media Marketing (SMM) aprueban la eficacia de los medios sociales para generar notoriedad de marca ${ }^{2}$. Como ejemplo, en Facebook, la empresa tiene un amplio abanico de posibilidades comunicativas: página corporativa, aplicaciones, anuncios sociales, historias patrocinadas, promociones y concursos, eventos, integración de la tienda virtual (conocida como $f$-commerce) y aprovechamiento de las oportunidades que brinda la geolocalización a través de Facebook Places.

De acuerdo con el estudio de IBM publicado en octubre de 2011 con el título From stretched to strengthened: insights from the global chief marketing officer study ${ }^{3}$, en opinión de los directores de marketing los medios sociales son la herramienta cuyo uso más va a crecer en los próximos tres a cinco años:

Cuadro 2. Tecnhology that CMOs worldwide plan to increase use of in the next 3-5 years (junio de 2011)

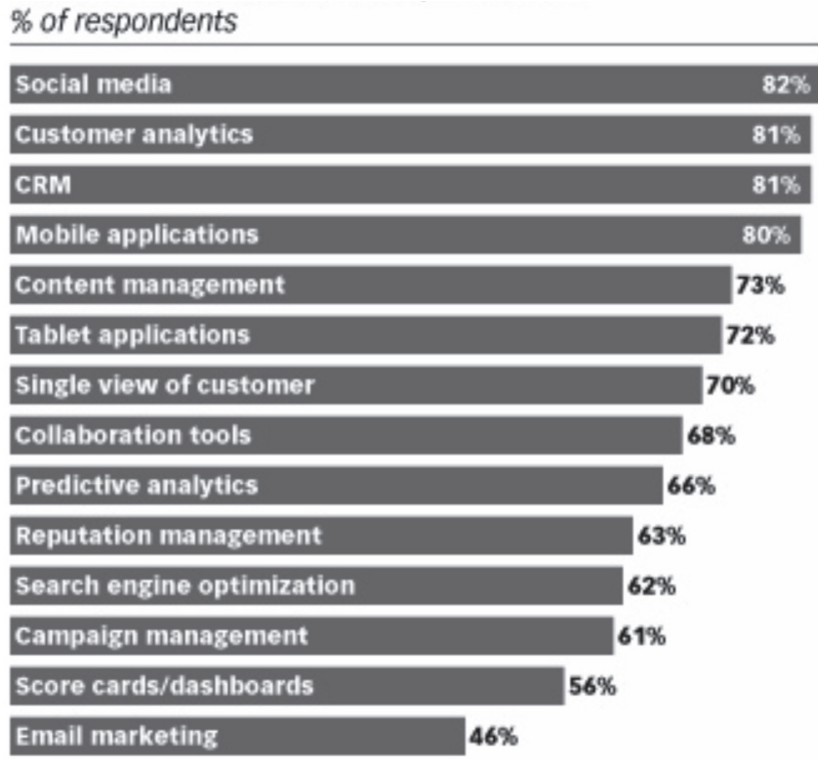

Es fundamental que las empresas sean conscientes de la necesidad de adaptar sus estrategias de comunicación empresarial y publicitaria a las peculiaridades

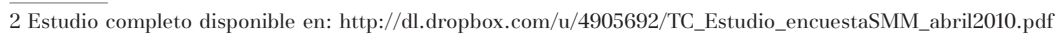

3 Disponible en www.emarketer.com 
de las plataformas digitales de manera profesional para aprovechar al máximo sus potencialidades y de llevar a cabo una comunicación integral e integrada entre acciones online y offline. Internet debe formar parte de la Integrated Communication Strategy (Victoria, 2005: 51), que lleva a la empresa a afrontar su labor comunicativa desde una visión global y holística.

\title{
2. La comunicación digital y los medios sociales
}

De Salas (2002: 72) se pregunta cómo ha transformado Internet la comunicación empresarial e institucional, a lo que responde:

\begin{abstract}
«Debido a la incorporación de la tecnología multimedia interactiva, la corriente o flujo informativo entre la empresa y sus posibles públicos se modifica. Los dos modos clásicos en que las empresas emiten los mensajes dirigidos a sus clientes y público externo (pull y push) toman valores y caminos diferentes. A nivel genérico, podríamos decir que la empresa pierde protagonismo en las relaciones comerciales en detrimento del consumidor. Éste, a su vez, y gracias a las nuevas tecnologías, puede desarrollar una actividad más activa en la búsqueda y análisis de información, así como en todos los procesos de la relación mercantil que desarrolla con la empresa. Por lo tanto, las actividades comerciales apoyadas en las nuevas tecnologías, permiten y fomentan la mayor participación del sujeto consumidor».
\end{abstract}

Scolari (2008: 78) sintetiza las particularidades de las nuevas formas de comunicación frente a las tradicionales en la transformación tecnológica (digitalización), la configuración muchos-a-muchos (reticularidad), las estructuras textuales no secuenciales (hipertextualidad), la convergencia de medios y lenguajes (multimedialidad) y la participación activa de los usuarios (interactividad). Además, destaca que la interactividad de los medios digitales genera una ruptura de las categorías que fundaban el proceso cultural (producción-emisor y consumo-receptor): «al participar en el control de los contenidos, el usuario de los medios interactivos termina por convertirse en parte de ese contenido.

Parafraseando a Mcluhan, podría decirse que en las nuevas formas de comunicación digital el usuario es el mensaje» (2008: 98). La digitalización de la comunicación no sólo ha modificado los canales, sino que ha alterado los contenidos, los medios, su producción y su consumo, como afirma Tomé (2011: 57): «todo gira en torno a un contenido vivo, que ellos mismos pueden afectar y que consumen a través del medio, soporte, dispositivo y formato de su elección».

Para el autor Celaya (2008: 84-88), existen varios factores que han transformado el modelo de comunicación digital del 1.0 al 2.0:

- $\quad$ incremento de clientes más inteligentes, formados y exigentes.

- $\quad$ avalancha de mensajes comerciales, con sobreabundancia de información y congestión publicitaria.

- nuevos líderes de opinión. La democratización de la comunicación, la inmediatez en la difusión del mensaje y la fusión de los roles emisor-receptor ha- 
cen que cualquiera pueda ser emisor de mensajes y, por tanto, convertirse en prescriptor, como los bloggers.

- $\quad$ pérdida de credibilidad de los medios tradicionales.

- clientes en red. Las nuevas tecnologías han desarrollado la creación de múltiples comunidades con los mismos gustos, tendencias u opiniones. Las empresas deben tener en cuenta este alto grado de interconexión entre clientes actuales y potenciales.

El desarrollo experimentado por las redes sociales ha acentuado estos cambios en la comunicación digital, al ser los espacios en los que el internauta cada vez más ocupa su tiempo conectado, como demuestra el estudio Media Metrix de la empresa de medición ComScore, con el crecimiento en el uso de estas plataformas a nivel mundial de marzo de 2007 a octubre de 2011. De acuerdo con este estudio, uno de cada cinco minutos de tiempo online está siendo empleado en redes sociales, frente a apenas un $6 \%$ a principios de 2007 . Además, las redes sociales llegan a un uso del $82 \%$ de la población conectada a Internet en el mundo (alrededor de 1,2 billones de personas en total):

\section{Cuadro 3. Horas al mes dedicadas a espacios en la Red (en billones) y total visitan- tes únicos (en millones) ${ }^{4}$}

* Time spent on Communications includes time spent on web-based Email and Instant Messengers.
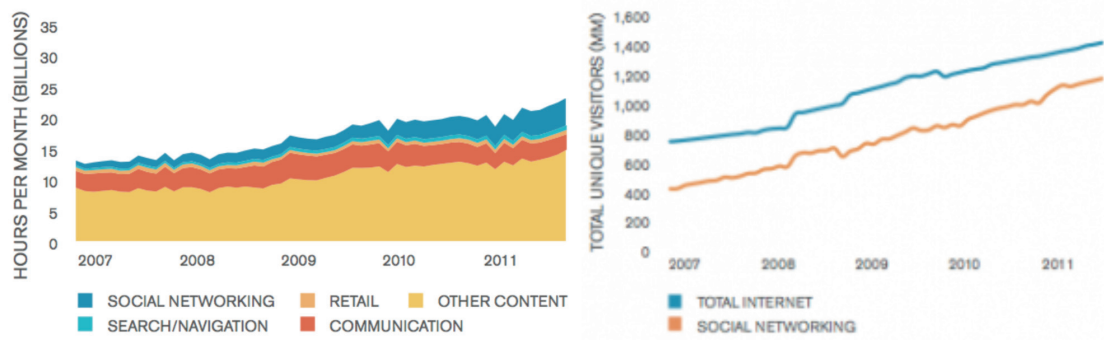

Este estudio también muestra el crecimiento experimentado en cuatro años por las principales redes sociales, siendo Facebook la más popular, con más de 800 millones de visitantes únicos:

\footnotetext{
4 http://www.comscore.com/Press_Events/Presentations_Whitepapers/2011/it_is_a_social_world_top_10_need-to-knows_ about_social_networking
} 
Cuadro 4. Total visitantes únicos (en millones) y tiempo (en meses) en alcanzar 25 millones de visitantes únicos
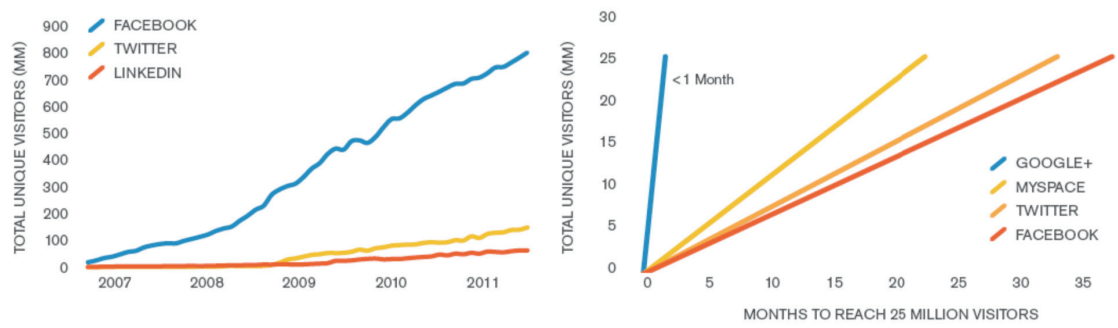

Otros estudios muestran las ventajas que, de acuerdo con los profesionales del sector, aportan los medios sociales en las estrategias de marketing, comunicación y publicidad. Según el estudio de Chief Marketer 2011 Interactive Marketing Survey, de mayo de 2011, la construcción de un vínculo que fidelice al consumidor actual o potencial es el principal beneficio de las campañas de Social Media Marketing ${ }^{5}$.

Cuadro 5. Principales beneficios de las campañas de SMM (Chief Marketer «2011 Interactive Marketing Survey»)

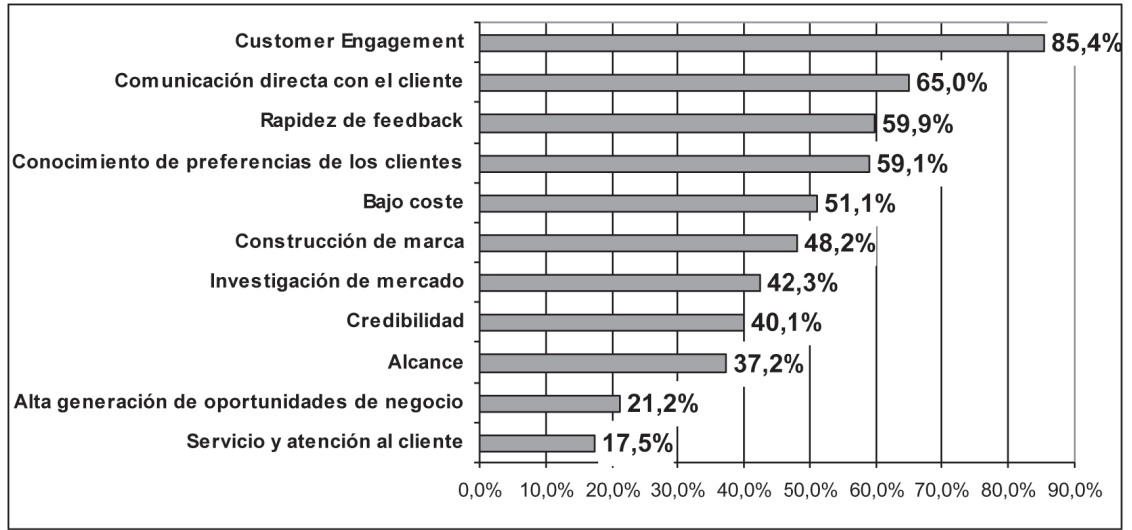

Pese a que todavía no se encuentran claramente definidos los parámetros más adecuados para la medición del retorno de la inversión (ROI) de las acciones de comunicación corporativa y publicitaria en medios sociales, la amplia variedad de indicadores de eficacia (Key Performance Indicators) para medir el ROI y, sobre

5 http://chiefmarketer.com/research/cm-interactive-survey-2011/ 
todo, el impacto en la relación (IOR) en las diversas plataformas sociales hace que los profesionales confíen en estos espacios como canales para una comunicación eficiente y rentable. Así lo destaca el estudio de MarketingSherpa 2011 Social Marketing Benchmark Report ${ }^{6}$, publicado en noviembre de 2011: si bien sólo el $20 \%$ de las agencias y consultoras de marketing y comunicación consultadas reconocieron que las campañas en medios sociales de sus clientes estaba produciendo ROI, el 64\% reconoció que sus clientes confiaban en que estos espacios darían sus frutos con el tiempo y estaban dispuestos a continuar invirtiendo en ellos:

Cuadro 6. Actitud con respecto a la capacidad de producir ROI de las actividades de Social Media Marketing

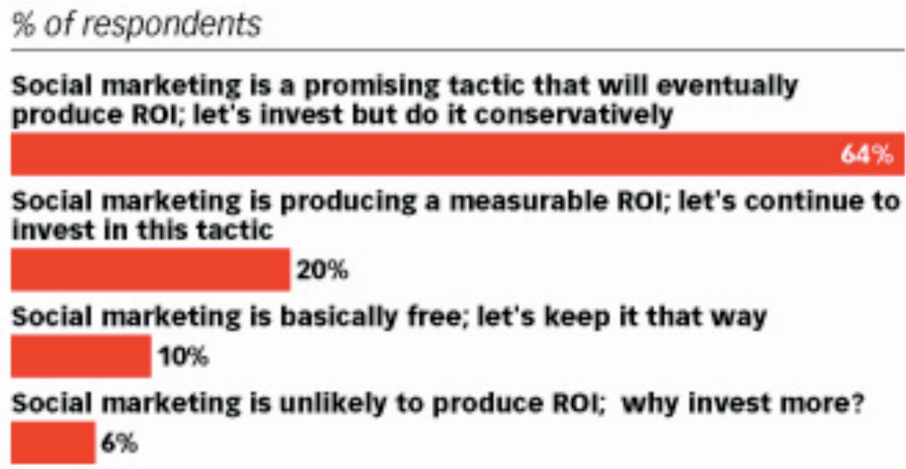

Hay que tener en cuenta que la complejidad que supone la construcción del vínculo con el usuario en los medios sociales escapa a la cuantificación objetivo de la fórmula del ROI, por lo que se requieren métricas más complejas que tengan en cuenta valores cualitativos como la conversación, la confianza, la influencia, el engagement, la prescripción y la participación. Así lo expresa Lamas (2010: 100):

«La medida de la efectividad de la comunicación a través de estas redes probablemente va a requerir nuevas métricas que incluyan, además de los clásicos indicadores de audiencia, otros factores más próximos al engagement, la influencia, la relevancia o la credibilidad».

Cuando el objetivo es humanizar a la marca y el posicionamiento ya no está en la mente, sino en el corazón (lovemark), como ocurre en los medios sociales, el retorno de la inversión está en el valor de cada cliente y se da en base a las relaciones que la marca construye con los usuarios.

En anteriores investigaciones se ha estudiado la gestión de la comunicación que la central de franquicia desarrolla para cada uno de sus tres principales públicos objetivo: los franquiciados actuales, los franquiciados potenciales y los clientes finales de sus establecimientos (Monserrat Gauchi, 2008). Esta peculiaridad de la franquicia, la existencia de un intermediario (el franquiciado, poseedor de la marca que comercializa) nexo de unión entre la central o casa matriz y los

6 Puede consultarse en www.emarketer.com 
clientes o consumidores finales del producto o servicio, hace que el uso de redes sociales se tenga que desarrollar con especial atención en este tipo de empresa. El objeto de estudio en esta investigación se aborda desde el conocimiento que en la central franquiciadora se debería tener para diseñar las estrategias de comunicación online teniendo en cuenta esta peculiaridad (la cesión de la marca e imagen a terceros, la estructura descentralizada de la empresa). Además, y como cualquier otro tipo de anunciante, el franquiciador debe tener en cuenta los cambios en el paradigma del sistema publicitario, tales como que el consumidor se está convirtiendo en emisor de mensajes sobre la marca, entre otros.

Por todo ello, si se tiene en cuenta que cada establecimiento adherido a la red puede gestionar la comunicación con su propio público objetivo y entorno, y en las diferentes redes sociales, este entramado comunicacional se complica todavía más.

\section{Hipótesis, objetivos y metodología}

Se parte de la hipótesis de que la empresa franquicia todavía tiene escasa presencia en los espacios 2.0 y, por lo que respecta a Facebook, se limitan a volcar información de otros canales de comunicación empresarial y publicitaria (página web, piezas publicitarias, etc.) en esta plataforma, generando poca conversación con sus seguidores.

El objetivo de este artículo se centra, pues, en la identificación de la presencia de una selección de empresas franquicia en un periodo de tiempo determinado en los principales entornos colaborativos de la Web 2.0, es decir, en el blog y en los medios sociales más importantes en términos de audiencia de acuerdo con los rankings de las empresas de medición Nielsen y ComScore, a saber: Facebook, Twitter, Youtube y Tuenti. Además, se revisan las comunicaciones llevadas a cabo por las franquicias de la muestra seleccionada a través de sus espacios en la principal red social, Facebook, con el fin de analizar la tipología de contenidos publicados y la interacción con los seguidores.

Junto con la investigación documental de publicaciones de los ámbitos profesional y académico relacionadas con la temática, la metodología se basa en un estudio exploratorio a partir del análisis de case studies de veinte empresas de franquicia españolas, extraídas del ranking Franchisa-30, que mide el progreso anual de las enseñas de franquicia a partir de los datos recogidos en la elaboración del Anuario Español del Franchising y del Comercio asociado, y del informe elaborado por la consultora especializada en franquicias Tormo \& Asociados 50 marcas líderes en franquicia, que incluye cadenas que por su reputación, dimensión y permanencia en el mercado han conseguido posicionarse por encima en sus segmentos de actividad ${ }^{7}$.

En una primera fase se revisa la presencia de las empresas franquicia analizadas en las plataformas 2.0 seleccionadas. Tras este primer análisis, se revisan los

7 Pueden leerse las noticias de ambos informes en: http://www.areafranquicia.com/sistema/ranking/index.asp?mnuYear=2010 y http://www.tormo.com/noticias/28145/Cuales_son_las_50_marcas_lideres_en_franquicia 
siguientes ítems en sus espacios en Facebook entre el 30 de noviembre y el 4 de diciembre de 2011:

- $\quad$ Número de publicaciones en el muro.

- Tipología de publicaciones en la página de Facebook: promociones y/o descuentos, información complementaria y/o noticias relacionadas, encuestas y/o preguntas a usuarios u otros.

- Resultados de las publicaciones en Facebook: número de «personas que están hablando de esto» , número de «me gusta», número de publicaciones compartidas por usuarios y número de comentarios de usuarios ${ }^{8}$.

- Respuestas a comentarios y dudas de usuarios, en caso de que existan.

- Otros contenidos de la página.

A partir del análisis de los resultados para cada uno de estos ítems y de la investigación documental del marco teórico se extraen las conclusiones para verificar o refutar la hipótesis inicial, basada en el escaso empleo de plataformas 2.0 por parte de la empresa franquicia y la limitada conversación generada con sus seguidores en la red social Facebook.

\section{Resultados}

En la siguiente tabla (cuadro 7) se presentan los resultados de la primera fase de la investigación, relativa a la presencia de las empresas franquicia que integran la muestra en los principales espacios 2.0.

De las 20 empresas analizadas, tres (15\%) son las que no cuentan con presencia corporativa en ninguna plataforma social (Bon Area, Yoigo K-Habls y Carlin), por lo que el $85 \%$ está presente en algún espacio 2.0. En el siguiente gráfico (cuadro 8) podemos ver el reparto de las 17 empresas franquicia restantes de acuerdo con el número de plataformas 2.0 con que cuentan.

8 Distinguimos entre los comentarios publicados por usuarios en las páginas en Facebook de los portales analizados y las publicaciones de la página que han sido compartidas por usuarios. 
Cuadro 7. La empresa franquicia en los medios sociales

\begin{tabular}{|c|c|c|c|c|c|c|c|}
\hline & & \multicolumn{5}{|c|}{ Plataformas 2.0} & \multirow[b]{2}{*}{$\begin{array}{c}\text { Total } \\
\text { plataformas }\end{array}$} \\
\hline & Franquicia & Blog & Facebook & Twitter & Tuenti & Youtube & \\
\hline 1 & Dia & 0 & 1 & 0 & 0 & 0 & 1 \\
\hline 2 & Vitalia & 0 & 1 & 1 & 0 & 1 & $\underline{3}$ \\
\hline 3 & Mc Donalds & 0 & 1 & 1 & 1 & 0 & 3 \\
\hline 4 & Carrefour Market & 1 & 1 & 1 & 0 & 0 & 3 \\
\hline 5 & Foster's Hollywood & 0 & 1 & 1 & 1 & 0 & 3 \\
\hline 6 & Bon Area & 0 & 0 & 0 & 0 & 0 & 0 \\
\hline 7 & Barceló Viajes & 0 & 1 & 1 & 0 & 0 & 2 \\
\hline 8 & Alain Afflelou & 0 & 1 & 1 & 1 & 1 & 4 \\
\hline 9 & Peggy Sue's & 0 & 1 & 0 & 0 & 0 & $\overline{1}$ \\
\hline 10 & Yoigo K-Habls & 0 & 0 & 0 & 0 & 0 & 0 \\
\hline 11 & Naturhouse & 0 & 1 & 1 & 0 & 1 & 3 \\
\hline 12 & Halcón Viajes & 1 & 1 & 1 & 0 & 0 & 3 \\
\hline 13 & Spar & 0 & 1 & 0 & 0 & 0 & $\overline{1}$ \\
\hline 14 & MRW & 1 & 1 & 1 & 0 & 1 & 4 \\
\hline 15 & Telepizza & 0 & 1 & 1 & 1 & 1 & 4 \\
\hline 16 & No+vello & 1 & 1 & 1 & 0 & 1 & 4 \\
\hline 17 & Carlin & 0 & 0 & 0 & 0 & 0 & 0 \\
\hline 18 & Gaes & 0 & 1 & 1 & 0 & 1 & 3 \\
\hline 19 & Vitaldent & 1 & 1 & 1 & 0 & 0 & 3 \\
\hline 20 & Marco Aldany & 0 & 1 & 1 & 0 & 0 & 2 \\
\hline & & 5 & 17 & 14 & 4 & 7 & 47 \\
\hline
\end{tabular}

Cuadro 8. $\mathrm{N}^{\circ}$ de plataformas empleadas

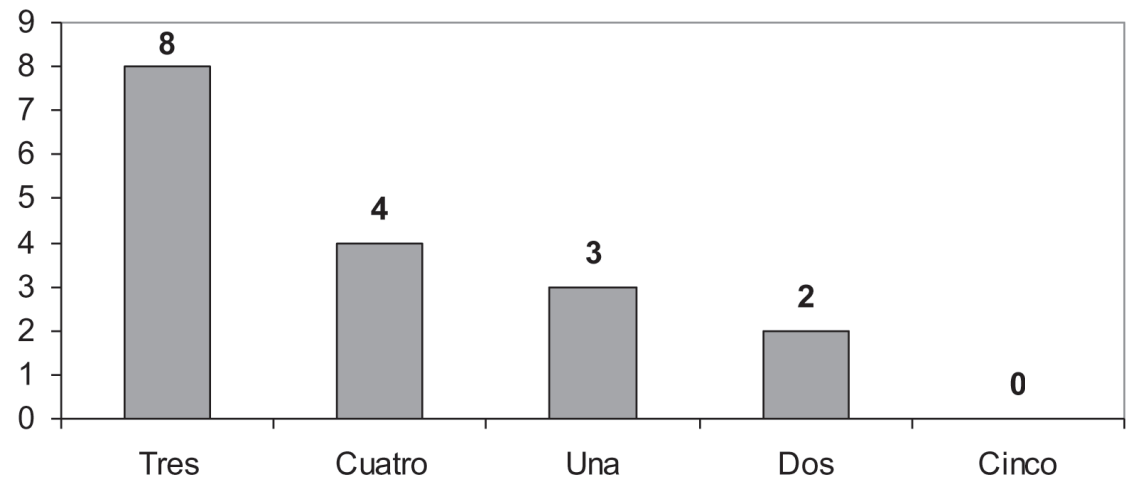


La mayoría de franquicias cuentan con tres y cuatro plataformas 2.0, siendo Facebook y Twitter las más populares, con 17 y 14 empresas, respectivamente. Es decir, el $100 \%$ de las empresas de la muestra que están presentes en espacios 2.0 tienen espacios en Facebook, siendo este porcentaje para Twitter del 82\%. Con respecto al resto de plataformas, Youtube ocupa la tercera posición en uso, seguido del blog y de Tuenti.

Ninguna de las empresas analizadas cuenta con espacios en las cinco plataformas 2.0 seleccionadas. Alain Affleou, MRW, Telepizza y Nomasvello son las empresas que tienen más plataformas, cada una de ellas con cuatro. En total, las 20 empresas de la muestra suponen 47 espacios en los entornos 2.0 analizados, lo que supone una media de 2,4 plataformas para cada empresa.

Pasando a la segunda fase del trabajo de campo, en el cuadro 9 se muestran los resultados a propósito de los contenidos de las 17 empresas franquicia que tienen presencia en la red social Facebook. Es importante tener en cuenta también la comunicación que la empresa franquicia hace en la página web de la existencia de estos espacios. En este sentido, siete de las 17 empresas que incluyen en las portadas de sus páginas web corporativas enlaces a sus espacios en entornos 2.0, como es el caso de Facebook (41\%).

Todos los espacios identificados en Facebook hacen referencia al ámbito geográfico español, a excepción de la página de Peggy Sue’s, que pertenece al establecimiento en Valencia, al no contar con página corporativa a nivel nacional.

De las 17 empresas con presencia en Facebook, el 65\% (11 franquicias) han publicado algún contenido en el periodo analizado de cinco días (del 30 de noviembre al 4 de diciembre de 2011). Entre las empresas que no han publicado nada, cabe destacar Dia y Spar puesto que, a pesar de contar con presencia en la red social, no cuentan con un muro en el que la organización y los usuarios puedan publicar, siendo la presencia por tanto meramente informativa. En las cuatro empresas restantes la última publicación es anterior al 30 de noviembre, incluso en algunos casos de hace meses, como sucede en la página de Barceló Viajes (la última publicación es del 25 de mayo de 2011). 
Cuadro 9. La empresa franquicia en Facebook

\begin{tabular}{|c|c|c|c|c|c|c|c|c|c|c|c|c|c|}
\hline & Franquicia & Página en Facebook & 1 & 2 & 3 & 4 & 5 & 6 & 7 & 8 & 9 & 10 & 11 \\
\hline 1 & Dia & /pages/Supermercados-Dia/103772662994996 & 0 & - & 23 & 1275 & - & - & - & - & - & - & - \\
\hline 2 & Vitalia & /GrupoVitalia & 16 & $p, i, o$ & 30 & 582 & 0 & 5 & 28 & 0,31 & 1,75 & 0 & $0 / 0$ \\
\hline 3 & Mc Donalds & /McDonaldsSpain & 5 & $p$, en & 3671 & 94616 & 0 & 572 & 459 & 114,40 & 91,8 & 5 & $0 / 0$ \\
\hline 4 & Carrefour Market & /carrefoures & 5 & $p, i$, en & 806 & 55084 & 0 & 144 & 168 & 28,80 & 33,6 & 18 & $19 / 19$ \\
\hline 5 & Foster's Hollywood & /fostershollywood & 7 & $\mathrm{i}, \mathrm{e}$ & 942 & 66414 & 26 & 50 & 269 & 7,14 & 38,43 & 1 & $25 / 25$ \\
\hline 6 & Barceló Viajes & /pages/Clubes-Barcelo-Viajes/156417437747844?sk=wall & 0 & - & 4 & 191 & 1 & - & - & - & - & - & 0 \\
\hline 7 & Alain Afflelou & /AlainAfflelouOptico & 2 & $\mathrm{p}$ & 63 & 13763 & 5 & 0 & 10 & 0 & 5,00 & 2 & $3 / 4$ \\
\hline 8 & Peggy Sue's & /pages/Peggy-Sues-Valencia/128605913878036 & 0 & - & 52 & 2259 & 0 & - & - & - & - & - & - \\
\hline 9 & Naturhouse & /Spain.NaturHouse?ref=ts & 0 & - & 42 & 2983 & 0 & - & - & - & - & - & - \\
\hline 10 & Halcón Viajes & /halconviajesonline & 12 & $p, i$, en, o & 143 & 8631 & 1 & 15 & 67 & 1,25 & 5,58 & 19 & $2 / 2$ \\
\hline 11 & Spar & /pages/SPAR/103802819658996 & 0 & - & 6 & 283 & - & - & - & - & - & - & - \\
\hline 12 & MRW & /mrwespana & 6 & $\mathrm{p}, \mathrm{i}$ & 110 & 4685 & 6 & 2 & 21 & 0,33 & 3,50 & 9 & $1 / 1$ \\
\hline 13 & Telepizza & /telepizza & 12 & $p$, i, en, o & 3613 & 262588 & 0 & 178 & 780 & 14,83 & 65,00 & 99 & $0 / 23$ \\
\hline 14 & No+vello & /nomasvellofotodepilacion?ref=ts & 9 & $p$, en & 108 & 4210 & 2 & 7 & 44 & 0,78 & 4,89 & 55 & - \\
\hline 15 & Gaes & /GAESCentrosAuditivos & 4 & $\mathrm{i}$, en & 83 & 2217 & 0 & 1 & 37 & 0,25 & 9,25 & 4 & - \\
\hline 16 & Vitaldent & /vitaldent & 0 & - & 156 & 625 & 0 & - & - & - & - & - & - \\
\hline 17 & Marco Aldany & /marcoaldany & 2 & $p, o$ & 73 & 4582 & 0 & 1 & 16 & 0,50 & 8 & 0 & - \\
\hline
\end{tabular}

1. Número de publicaciones en el muro entre el 30 de noviembre y el 4 de diciembre de 2011.

2. Tipología de publicaciones: promociones y/o descuentos $(p)$, información complementaria y/o noticias relacionadas (i), encuestas y/o preguntas a usuarios (en) u otros (o).

3. Número de "personas que están hablando de esto" a fecha 04/12/2011.

4. Número de "Me gusta" a la página a fecha 04/12/2011.

5. Número de publicaciones de usuarios entre el 30 de noviembre y el 4 de diciembre de 2011

6. Numero de comentarios de usuarios entre el 30 de noviembre y el 4 de diciembre de 2011 .

7. Número de "me gusta" a las publicaciones entre el 30 de noviembre y el 4 de diciembre de 2011.

8. Media de comentarios de usuarios para cada publicación en el periodo analizado.

9. Media de "me gusta" para cada publicación en el periodo analizado.

10. Número de veces que las publicaciones de la página del periodo analizado han sido compartidas por usuarios.

11. Ratio de respuestas a publicaciones y comentarios de usuarios con quejas y/o dudas en el periodo analizado. El guión (-)

indica la no existencia de quejas y/o dudas entre las publicaciones y comentarios de los usuarios en el periodo analizado.

Grupo Vitalia (16), Telepizza (12) y Halcón Viajes (12) son las empresas que superan las decena de publicaciones en el periodo analizado. En total, las $17 \mathrm{em}$ presas de la muestra suman 80 publicaciones en el periodo de cinco días, lo que supone una media de 16 publicaciones diarias y 4,7 publicaciones por empresa.

Con respecto al punto 2 de la tabla del cuadro 9, referente a la tipología de publicaciones, en general, promociones, ofertas y descuentos son los principales protagonistas de las publicaciones de las franquicias en Facebook, al aparecer en nueve de las once empresas con publicaciones en el periodo analizado (82\%), si bien información complementaria o noticias relacionadas y preguntas a usuarios son también bastante populares, con siete casos cada una (64\%). Un ejemplo de ello es Vitalia, muy activo en la página de Facebook y con contenidos que aportan valor añadido al usuario. 
Cuadro 10. Ofertas, descuentos y promociones en las publicaciones en Facebook

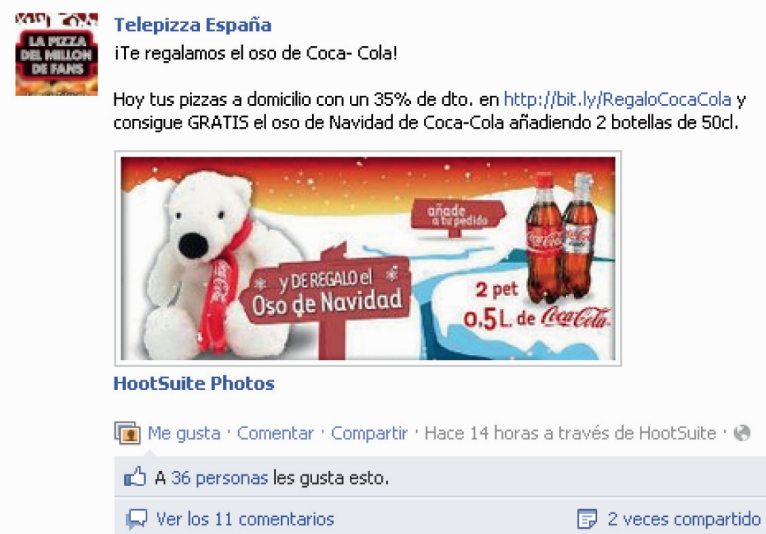

\section{Cuadro 11. Noticias relacionadas y preguntas en las publicaciones en Facebook}

Buen fin de semana a todos;-)

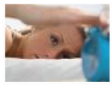

La dieta mediterránea combate problemas de sueño whw muyinteresante,es

Muy Interesante. La dieta mediterránea combate problemas de sueño. Salud en Muy Interesante: noticias, artículos, entrevistas, curiosidades, imágenes y videos sobre salud.

207 Me gusta - Comentar ' Compartir ' El Viernes a las 14:45 - a

Grupo Vitalia

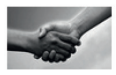

¿̇́ómo potenciar la autonomía de las personas mayores dependientes?

- Acondicionar el entorno de la persona mavor para facilita conductas independientes, - Observar a le persona mayor para conocer todo lo que puede hocer por simisma may

口 Me gusta ' Comentar ' Compartir · El Viernes a las 13:54

¿3 A Raquel Luis le gusta esto.

VITAuri. Grupo Vitalia Mañana es el Día Mundial de las personas con discapacidad. Por ello pensamos que estos pueden ser unos útiles consejos para mucha gente. IEsperamos que os sirvan! :) El viernes a las 13:55 ' Me gusta

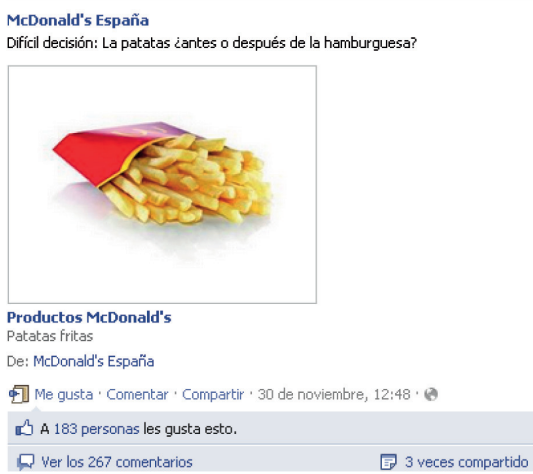

Escribe un comentario...

En cuanto al valor «número de personas que están hablando de esto», destacan Telepizza y McDonalds, con más de 3000 personas, seguidas de Foster's Hollywod y Carrefour, con 942 y 806, respectivamente. En número de fans, es Telepizza la empresa franquicia de la muestra más popular, con 262.588 fans, seguida de McDonalds (94.616 fans), Foster's Hollywood (66.414 fans), Carrefour (55.084 fans) y Alain Affleou (13.763 fans). El resto de empresas franquicia no llegan a los 10.000 fans. En general, las empresas más populares coinciden con 
las que publican con más asiduidad. Además, McDonalds, Carrefour, Foster's Hollywood y Peggy Sue's tienen integrado Facebook Places, que permite a los usuarios hacer check-in, es decir, indicar que «estuvieron aquí». En el caso de McDonalds, 33.683 personas hicieron check-in.

Si calculamos el ratio entre «personas hablando de esto» y el número de «me gusta», Vitaldent es la empresa con más relevancia, al tener un ratio de $25 \%$, seguida de Vitalia, con 5,2\%. El resto de empresas tiene ratios inferior a los cinco puntos.

El valor 5 de la tabla de resultados hace referencia al número de publicaciones de los usuarios en los muros de Facebook de las empresas franquicia de la muestra en el periodo analizado. Únicamente seis empresas tienen valores en esta columna, siendo el de Foster's Hollywood el muro en el que los usuarios más publican.

En general, se trata de quejas de usuarios: «En el Foster de Kinépolis de Granada es imposible comer, hace más frío que en Groenladia y la calefacción apagada, con lo que cobran por la comida...», "Dadle un toque a los del restaurante del parque corredor en Torrejón, Madrid. Tener que pedir los cuchillos, no poner los platos individuales hasta el segundo plato, traer el primero y a los 5 minutos el segundo, la música ensordecedora, ni un camarero pendiente de la mesa salvo los de bebidas, pedir ensalada de col y traer cualquier otra cosa rara que era aquello... un fiasco. Cada vez va peor el servicio».

Los usuarios también suelen publicar preguntas, como por ejemplo: « ¿hay alguna promoción para este miércoles de cheque gorrón?», "Las costillas que hacéis o las alitas de pollo... están hechas a la brasa siempre con salsa?», « ¿Tienen en MRW un envío gratis para familia numerosa?». En dos ocasiones, en los muros de Telepizza y MRW, los usuarios han publicado búsqueda de empresa.

Pasando a los comentarios de los usuarios en respuesta a las publicaciones de la empresa franquicia en el muro de Facebook, McDonalds, Telepizza y Carrefour son las únicas que sobrepasan el centenar de comentarios, con 572, 178 y 144, respectivamente. Publicaciones de McDonalds como «¿Con qué bebida sabe mejor tu hamburguesa?» y «Difícil decisión: las patatas fritas ¿antes o después de la hamburguesa?» consiguen 174 y 267 comentarios. Foster's Hollywood suma 50 comentarios en los cinco días analizados. La media total de comentarios a las publicaciones recogidas en la muestra de empresas franquicia asciende a 12,19 comentarios, al haber 975 comentarios para 80 publicaciones. Por encima de esta media se encuentran McDonalds, con una media de 114,4 comentarios para las cinco publicaciones, Carrefour con un 28,8 y Telepizza con 14,83 (valores representados en la columna 8 de la tabla de resultados).

Los «me gusta» indicados por los usuarios en respuesta a las publicaciones de la empresa franquicia en el muro de Facebook ascienden en general a 1.899, lo que supone una media de 23,8 para cada publicación. Telepizza, McDonalds 
y Foster's Hollywood son las empresas con mayor número de «me gusta», con 780, 459 y 269, respectivamente. Junto con estas tres empresas, Carrefour también supera la media de «me gusta» por publicación, con 33,6 para cada una de ellas. El resto no supera el valor de 23,8.

Además de los comentarios y los «me gusta», una forma de interacción de los usuarios en respuesta a las publicaciones de la empresa en el muro de Facebook son las acciones de compartir los contenidos con sus contactos. Sin embargo, no se trata de una acción muy popular, ya que es de las tres la que menos realizan los usuarios: en total, las 80 publicaciones son compartidas 212 veces, lo que representa una media de 2,65 veces compartidas para cada publicación y de 19 para cada una de las once empresas que han publicado en el muro de Facebook en el periodo analizado. No obstante, esta última media se encuentra sesgada por el muro de la empresa franquicia Telepizza, que es la que más «veces compartido» recibe, con 99.

En total, los tres tipos de interacción del usuario (comentarios, «me gusta»y veces compartido) suman 3.086 interacciones para las 80 publicaciones, por lo que la media asciende a 38,6 interacciones para cada publicación y 281 interacciones para cada una de las once empresas con publicaciones, valores que podemos considerar elevados.

De los resultados anteriores podemos concluir que McDonalds, Carrefour, Foster's Hollywood y Telepizza son las empresas más populares por las interacciones de sus usuarios. En estos espacios los usuarios interactúan con frecuencia y son activos ante preguntas como «Si tengo carne 100\% vacuno, lechuga, cebolla, pepinillos, queso y salsa, todo ello dentro de pan con semillas de sésamo... ¿Quién soy?», con 80 comentarios. En el caso de Carrefour, los usuarios suelen incluir comentarios con dudas sobre las promociones y en los muros de Telepizza y Foster's Hollywood expresan sus experiencias con el servicio a domicilio o el trato recibido en el local, respectivamente, tanto positivas como negativas. Los usuarios incluso en ocasiones actúan como fansumers defendiendo a la marca ante algún comentario negativo de otro usuario: «Yo hice un pedido a las 8:55 pm y la telepizza llegó a las 9:15 pm (menos de media hora). Me llevaron todo lo que les pedí, con un gran sabor y buen precio. Me he ahorrado el metro y el tiempo para ir a comer a otro lugar. Si ha usted le parece caro... simplemente no compre, búsquese un buen trabajo, cocine en su propio hogar y deje de quejarse como si fuese un niño».

Foster's Hollywood, Telepizza y Carrefour son las empresas que más preguntas de usuarios reciben, con 25, 23 y 19, respectivamente. Mientras que Foster's Hollywood y Carrefour responden al 100\% de las quejas, dudas o reclamaciones, Telepizza aporta a las consultas de los usuarios poco feedback. La interacción que aporta la empresa al usuario ante sus preguntas y/o quejas se muestra con comentarios como «Lamentamos la experiencia que has tenido. Si no te importa, podrías hacerlo por escrito en el vínculo que te dejamos. Es la forma más efi- 
caz de que tus comentarios sean monitorizados, se siga el problema y podamos resolverlo satisfactoriamente» o «Agradecemos tu comentario y desde luego lo tendremos muy presente», ambas de Foster's Hollywood.

Por último, comentamos otros aspectos relevantes de la presencia en Facebook de las empresas franquicias de la muestra analizada. En concreto, destaca Telepizza por ser la única empresa que cuenta con plataforma de comercio electrónico integrada en la red social. El comercio electrónico a través de Facebook se conoce como $f$-commerce y hace uso de aplicaciones de tiendas virtual que quedan integradas dentro de la red social (como Payvment), anuncios de los productos en el muro, publicación de catálogos, etc. La tienda integrada de Telepizza en Facebook es el caso más conocido en España, con descuentos exclusivos si se realiza el pedido en este canal. MRW España no incluye la posibilidad de contratación del servicio en Facebook pero sí incluye una pestaña dedicada a su plataforma de comercio electrónico externa a la red social.

Otros contenidos que se incluyen en los espacios de las empresas franquicia en Facebook son la aplicación encuestas, empleada por McDonalds, Carrefour, Foster's Hollywood o Halcón Viajes, con preguntas a usuarios a propósito de sus productos o servicios. Las empresas también añaden pestañas dedicadas a promociones puntuales (como la acción Monopoly de McDonalds) y servicios específicos (por ejemplo, transporte para el sector sanitario en el caso de MRW España).

La geolocalización mediante la integración de Facebook Places, en el caso de McDonalds, la inclusión de normas sociales para el uso de la página en Facebook (casos como Telepizza y Foster's Hollywood) y la integración con otras plataformas como Twitter y Youtube, como en el caso de Nomásvello, son otros contenidos incluidos en los espacios de las empresas en la red social.

Los programas de Responsabilidad Social Corporativa también se incluyen en los contenidos de las empresas en Facebook. MRW España cuenta con una pestaña dedicada a la Fundación Red de Emociones y a las campañas solidarias de marketing con causa y Telepizza también dedica una pestaña a campañas solidarias con motivo de las Navidades.

Gaes es una de las empresas que tiene la página en Facebook más completa, con pestaña de bienvenida, pestaña para ofertas de trabajo, encuestas, buscador de centros, test auditivo o aplicaciones como «envía un sonido» (cuadro 12).

En general, todas las empresas que tienen presencia en la red social emplean en sus comunicaciones un lenguaje informal, coloquial y cercano, siendo habitual el empleo de emoticones y exclamaciones para enfatizar lo que se transmite. 


\section{Cuadro 12. Página en Facebook de Gaes}
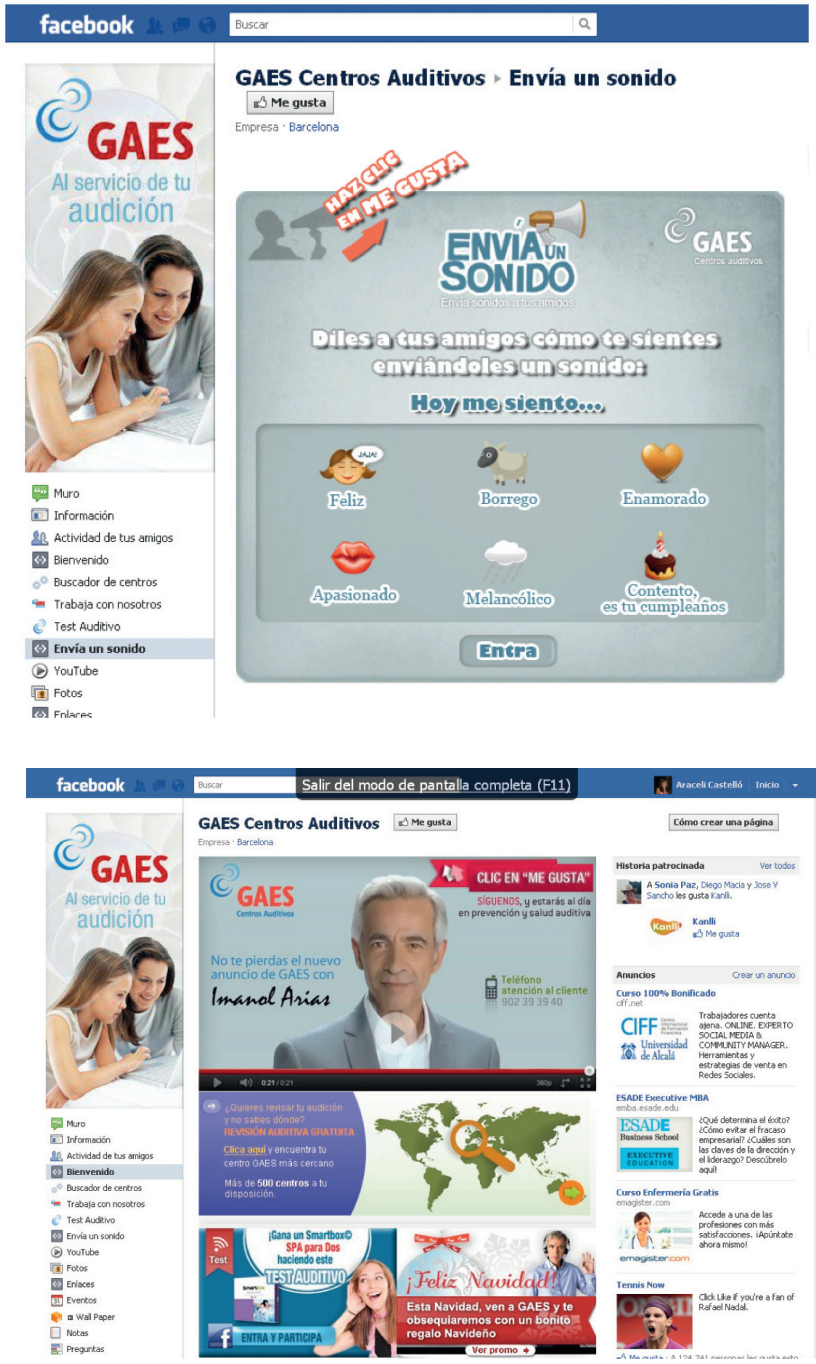


\section{Conclusiones}

Los medios sociales se han convertido en un canal de atención al cliente y venta en el que la empresa busca lograr conversión, fidelidad y recomendación. La creación y el establecimiento de un vínculo con el usuario a través de aspectos como los que hemos analizado, a saber, la generación de contenidos relevantes y de valor y la interacción con el usuario, son claves para el éxito en las plataformas sociales. Como destaca Tomé (2011: 189), «el marketing en redes sociales no incrementa directamente las ventas, pero crea embajadores que generan recomendaciones».

Para poder involucrar al usuario y desarrollar experiencias personalizadas, afines y relevantes para el consumidor es necesario que la empresa participe en la conversación, como resultante de una estrategia definida que involucre a toda la organización, y que sea consciente de la importancia que adquieren los contenidos de valor como necesidad competitiva.

Sin embargo, sin ánimo de concluir por la dimensión de la muestra, los resultados del estudio aportan indicios de un escaso aprovechamiento por parte de la empresa franquicia de las bondades de las plataformas 2.0. Si bien la empresa franquicia, en general, tiene presencia en medios sociales, las comunicaciones analizadas muestran una gestión lejana a la filosofía 2.0, ya que el usuario sí interactúa con la empresa con asiduidad (con comentarios, «me gusta» y veces compartido) pero ésta en pocas ocasiones continúa la conversación y, en general, aporta reducida retroalimentación a los seguidores. Además, espacios como Twitter requieren un manejo eficiente de los tiempos de respuesta al usuario por la inmediatez que les caracteriza como canal de atención al cliente. Las promociones y descuentos se utilizan como gancho para captar usuarios en estas plataformas, pero no son suficientes para fidelizar al usuario, que busca también contenidos de valor añadido (noticias relacionadas, aplicaciones, información relacionada con el desarrollo sostenible, etc.). Las preguntas a los usuarios son una de las publicaciones que más fomentan la interacción de los seguidores, empleando un lenguaje cercano y un estilo de comunicación informal. La correcta gestión de los comentarios negativos es uno de los retos para las empresas con presencia en medios sociales, en los que los usuarios exponen de manera pública sus quejas en base a su experiencia de compra y consumo.

Por tanto, si la empresa franquiciadora quiere aprovechar las bondades que, desde el punto de vista de las estrategias empresariales, pueden ofrecerle las plataformas de la Web 2.0, deberá llevar a cabo un planteamiento de sus canales de comunicación mediante la incorporación de estos nuevos espacios, en base a una planificación estratégica, y gestionar su reputación online de forma profesional, mediante perfiles como el del community manager. Por otro lado, es de vital importancia la correcta gestión de los espacios en los que aparece la marca-franquicia en las redes sociales. Es importante que el consumidor sepa de quién se trata, de si está contactando o uniéndose a una red social de 
la central de franquicia, o de un establecimiento franquiciado. En este sentido proponemos una estrategia clara y definida a desarrollar desde la central de franquicia que determine la presencia de cada uno de los franquiciados en las redes sociales. Dado que se comparte una marca e identidad común es de vital importancia realizar esta diferenciación para que cada usuario sepa con quién está hablando en cada momento.

\section{Bibliografía}

Celaya, Jesús (2008). La empresa en la Web 2.0. Madrid: Gestión 2000.

De Salas Nestares, María Isabel (2002). La comunicación empresarial a través de Internet. Valencia: Servicio de publicaciones Universidad CEU-Cardenal Herrera.

Lamas, Carlos (2010). Los medios interactivos y su publicidad. La medición de audiencias. En: Telos, $\mathrm{n}^{\mathrm{O}}$ 82. Disponible en

http://sociedadinformacion.fundacion.telefonica.com/DYC/TELOS/SOBRETELOS/Nmerosanteriores/DetalleAnteriores_82TELOS_DOSSIERPV1/ seccion $=1268 \&$ idioma $=$ es_ES $\& i d=2010020409130001 \&$ activo $=6$.do. Consultado el 17/06/2011.

Monserrat-Gauchi, Juan (2008). Análisis comparativo de las estrategias de Comunicación en franquicia. Enseñas consolidadas versus emergentes. En: Revista Latina de Comunicación Social, $\mathrm{n}^{\mathrm{0}}$ 63. La Laguna: Universidad de La Laguna, 106-120. Disponible en:

http://www.revistalatinacs.org/_2008/09_Alicante/Juan_Montserrat.html. Consultado el 20/09/2011

Scolari, Carlos (2008). Hipermediaciones. Elementos para una teoría de la comunicación digital interactiva. Barcelona: Gedisa Editorial.

Tomé, Pepe (2011). Conecta! La empresa en la red social. Barcelona: Libros de Cabecera.

Victoria Más, Juan Salvador (Coord.) (2005). Reestructuras del sistema publicitario. Barcelona: Ariel Comunicación.

\section{Referencia de este artículo:}

Castelló Martínez, Araceli y Monserrat Gauchi, Juan (2012). La comunicación digital en la empresa de franquicia: uso y presencia en los medios sociales. En: adComunica. Revista Científica de Estrategias, Tendencias e Innovación en Comunicación, $\mathrm{n}^{0} 3$. Castellón: Asociación para el Desarrollo de la Comunicación adComunica, Universidad Complutense de Madrid y Universitat Jaume I, 105124. DOI: http://dx.doi.org/10.6035/2174-0992.2012.3.7 